\title{
Design of Defecation Parameter Monitoring System Based on the Internet Plus
}

\author{
Lijun Hao*, Chunyu Shan and Jiayong Yan \\ Shanghai University of Medicine \&Health Sciences, Shanghai, China \\ ${ }^{*}$ Corresponding author
}

\begin{abstract}
A defecation monitoring system based on weighing sensor with Internet Plus was designed in the paper. In the system, 8051 single chip is applied as a control core. First of all, users' defecation information is collected by some weight sensors. And then, the information about change in weight that is analog is converted to digital signal by an $\mathbf{A} / \mathbf{D}$ conversion chip. After that, the digital information is transmitted to 8051 and is analyzed furthermore. Finally, these defecation parameters are stored and are further uploaded to the cloud server via WIFI for easy access by family doctors. Compared with the traditional monitoring, such as ECG and blood pressure, this system selects defecation parameters which can reflect the status of human metabolic system as monitoring information. It has a certain clinical significance for the detection of gastrointestinal chronic diseases. In addition, by combing with the Internet plus, it is contribute to the integration with cloud medical system in the future and can provide real-time monitoring for elderly.
\end{abstract}

Keywords- monitor system; defecation parameter; weight sensor; internet plus

\section{INTRODUCTION}

The aging population in our country is becoming more and more serious, and the health of the elderly has become a major social issue. At present, the health status of the elderly is monitored by routine clinical monitoring parameters, such as blood pressure, body temperature, respiration, ECG and blood sugar. These parameters are mainly related to heart, respiratory, endocrine and other systems [1, 2]. However, the description of the situation of human metabolic system, such as defecation monitoring, are hardly referred.

Bowel movements come from the intestines. They are just like mirrors, can reflect the conditions of the intestines and learn about our health. Generally, it would be impossible for a person to observe the bowel condition in daily life without a detailed bowel examination in the hospital. In fact, our health is greatly affected by the intestinal environment [2]. Therefore, we can be aware of our health to some extent by monitoring daily bowel movements [3].

Just like the weather experts can judge the weather condition according to the snowfall at that time. We can start with the "excretion"-defecation frequency and defecation rate when we want to detect bowel movements [4]. The clinical manifestations included prolonged defecation time, difficulty in defecation, decreased defecation frequency or defecation, and irregular defecation [2].

The information, we call them as the defecation parameters. These parameters cannot be measured directly in everyday life.
The present invention measures these parameters by indirect methods, i.e., by measuring body weight and its rate of change, these parameters are detected.

\section{SYSTEM DESIGN}

In order to achieve these defecation parameters, a defecation monitoring parameter system was designed, as shown in figure 1.
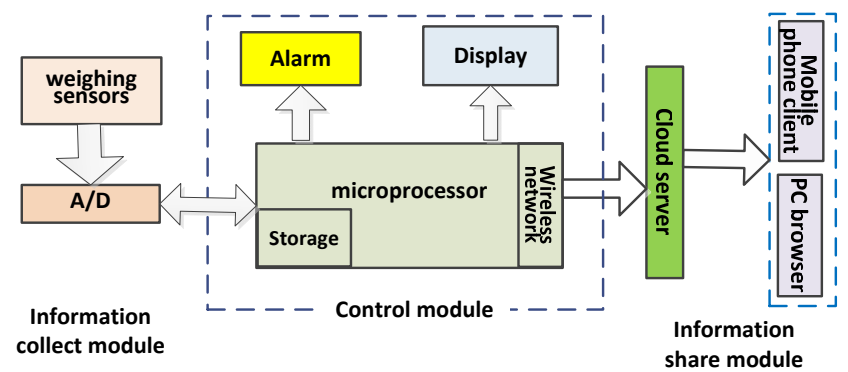

FIGURE I. STRUCTURE OF DEFECATION PARAMETER MONITORING SYSTEM

As shown in Figure I, the system consists of an information collect module which is composed of some weighing sensors and an A/D conversion chip, a control module, a cloud system and some clients that forms an information sharing module [5].

In the information collect module, weighing sensors are applied to obtain the weight change and indirectly measures the amount of excretion [6]. In the system, four sensors are placed in in the four corners of the stool. The weight can be achieved by accumulating these results when the users sit on the stool at any time. Then the output of weighing sensors that is analog signal, is converted to digital output signal after $\mathrm{A} / \mathrm{D}$ conversion that can be processed by 8051 single chip [7].

In the control module, the digital signals can be processed and analyzed furthermore. The system monitors the weight change value by the weighing sensors and indirectly measures the amount of discharge. When the measuring value exceeds the normal value, the alarm or prompt can be sent out. Moreover, wireless transmission module and memory module is respectively connected the microprocessor. The analysis results can be stored in the local computer.

Meanwhile, the parameters can be further sent to the cloud server by a wireless transmission module to provide the basis for the daily monitoring of the physiological status of the elderly by doctors or guardians $[8,9]$. Doctors or families can 
obtain the monitor result by browsing web or APP easily. This part, here we name it information share module.

In addition, the weighing sensors are arranged in the bottom of the chair legs, and the control module is electrically connected with the weighing sensor.

\section{MAIN TECHNOLOGY}

\section{A. Information Acquisition}

The main purpose of this system is to monitor and record the situation of toilet in real time. The weight of the human body will change before and after going to the toilet. The value can be measured by a weighing sensor symmetrically placed in the four corners of the stool chair [5, 6, and 8].

The module for collecting weight information is shown in Figure II. The double cantilever beam parallel strain weighing sensor (CZL-601 type weighing sensor) are adopted as the weighing sensors. CZL-601 type weighing sensor is characterized by high accuracy, easy processing, simple and compact structure, strong anti-bias capacity, high natural frequency, CZL-601 accuracy of + 0.02\%F.S, sensitivity of $2+$ $0.1 \mathrm{mV} / \mathrm{V}$, range of $40 \mathrm{~kg}$.

\section{Collect weigh information}

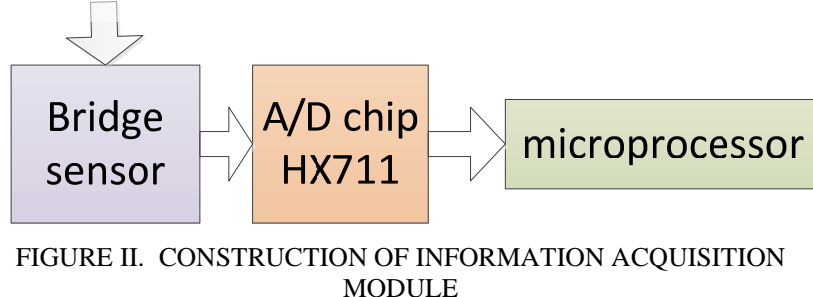

The A/D chip uses a high precision A/D conversion chip, such as HX711 type as an A/D converter chip [10].

The HX711 A/D conversion chip is a 24 bit A/D converter chip designed for high-precision weighing sensors. Compared with other types of chip, the chip peripheral circuit includes a voltage stabilized power supply, on-chip clock oscillator with other types of chips needed, with high integration, fast response speed and strong anti-jamming etc... The whole cost of the electronic scale is reduced, and the performance and reliability of the whole machine are improved. The interface and programming of the chip and the back-end microprocessor chip are very simple. All the control signals are driven by the pins without programming on the chip's internal registers. The input selector switch can optionally select channel A or channel B, which is connected with the internal low noise programmable amplifier. The programmable gain of the channel $A$ is 128 or 64 , and the corresponding full differential input signal amplitudes are $+20 \mathrm{mV}$ or $+40 \mathrm{mV}$. The channel B is a fixed 32 gain for system parameter detection. The power supply provided in the chip can directly supply power to the external sensor and the A/D converter in the chip, and no analog power source is needed on the system board. The on-chip clock oscillator does not require any external devices. The power on auto reset function simplifies the initialization process of the boot.

\section{B. Signal Processing}

After the weighing sensors collect weight information data, the data is transmitted to HX711. Then the digital weight change information is processed by 8051 .

The control module adopted 8051 single-chip as a control core, records the defecation parameters in real-time. The software flow is shown in Figure III.

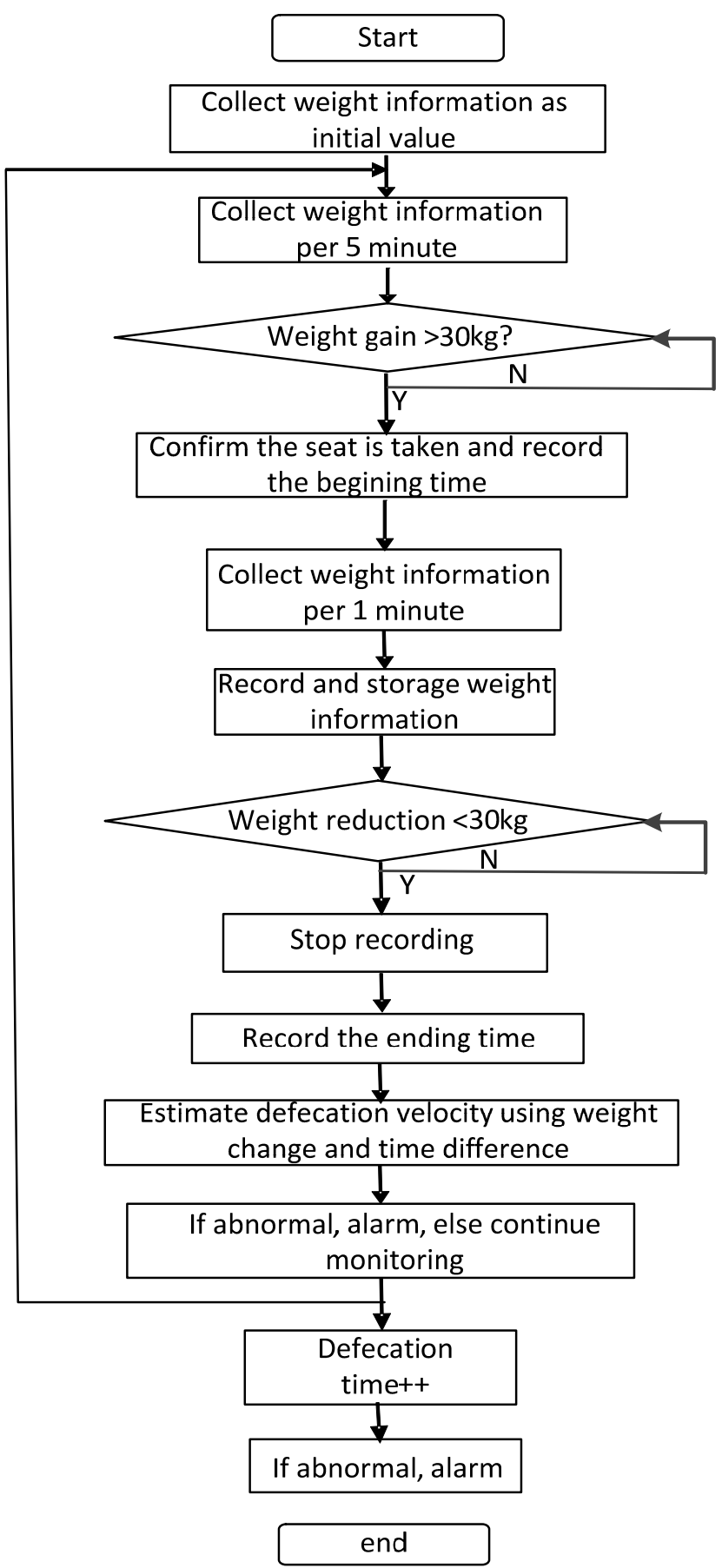

FIGURE III. SOFTWARE FLOW 
By the model, these results can be processed and screened. Furthermore, if there is some abnormal cases, the system will alarm at the same time.

Later the analyze results will be stored in the memory and will be uploaded to the cloud server through the wireless transmission module at the same time. Finally the information will be transmitted to the remote terminal.

\section{Information Share}

Information on the cloud server can be shared with third parties, such as micro-blog and WeChat, which can also be accessed by mobile phone users via APP. This is conducive to the elderly in the home crisis occurs, the information sent to the relevant guardian of the mobile phone on time, so that community doctors and families can quickly feedback. In addition, historical information helps doctors to monitor patients more carefully and effectively.

\section{APPLICATION}

Based on the weighing sensors,the system designed in the paper can monitor the user's toilet information in real time. Combing with the Internet Plus, doctors or families can visit the cloud server anytime or anywhere by browsing the web or APP of the mobile terminal, and check the user's information about the toilet, which is more effective in detecting some chronic diseases. Compared with the traditional monitoring of ECG and blood pressure information, this system selects defecation parameters which can reflect the status of human metabolic system as monitoring information, and has a certain clinical significance for the detection of gastrointestinal chronic diseases. In addition, the application of cloud system, will be contribute to the integration with cloud medical system in the future, to provide real-time monitoring for the elderly [11].

Figure III illustrates the software flow. Here, we will introduce the work stages of the system more detail. The mainly stages includes 7steps.

Step 1, turn on the power switch of the control module and make the system start working;

Step 2, the microprocessor of the control module receives the weight information transmitted by the A/D conversion chip for the first time and uses it as an initial value;

Step 3, the A/D conversion chip collects the weight information of the weighing sensor every 5 seconds, and transmits the secondary weight information to the microprocessor;

Step 4, the microprocessor will weight information and initial step three values were collected by comparison and analysis, determine the weight increase value is greater than 30 $\mathrm{kg}$, if more than, to determine who has sat on the toilet seat, and to record the start time of defecation;

Step 5, A/D conversion chip start every 1 seconds once the weight of the weighing sensor acquisition information, and the weight information is transmitted to the microprocessor; the microprocessor the weight information and the weight information of a comparison analysis, determine the weight reduction value is greater than $30 \mathrm{~kg}$, if less than this, the weight information is stored to the storage module and if more than, is judged to have left the toilet chair, storage data and record the end time of defecation;

Step 6, the microprocessor uses the weight change and the time difference to estimate the defecation speed and the defecation frequency and so on. If there is an abnormality, the alarm prompt module is started, otherwise the standby is carried out until the next monitoring starts;

Step 7, the cumulative microprocessor will store the monitoring data in storage within the module through the wireless transmission module to upload to the cloud server, and then transmitted to the remote terminal; the remote terminal analysis of monitoring data, if there is abnormal, then sends the preset warning information, otherwise, waiting for the next analysis.

\section{CONCLUSION}

The aging population in our country is becoming more and more serious, and the health of the elderly has become a major social issue. The defecation parameters are closely related to human metabolic system. However, there is almost no system for monitoring defecation parameters. The present telemedicine monitoring system is mainly used to monitor the physiological status of patients, such as blood pressure, body temperature, respiration, ECG, blood sugar and so on which related to human metabolic system. In the paper, a real-time monitoring system for defecation parameters is designed, which can also be connected to the Internet and transmitted to the cloud system in real time. The guardian or community doctor can access the defecation parameters of the user through mobile phone access, providing an auxiliary basis for effective monitoring of the patient. The design of this system can not only save the cost effectively, but also facilitate the management of information. It is beneficial to integrate with the future cloud medical system. The application of this system can help to understand the daily state of the elderly and find out the abnormal state, so as to improve the quality of life of the elderly.

\section{REFERENCES}

[1] D. Sikirov, "Comparison of straining during defecation in three positions: results and implications for human health". Digestive Diseases and Sciences, Vol.48, pp.1201-1205, 2003.

[2] WP Voskuijl, HJ van der Zaag-Loonen, IJ Ketel, MA Grootenhuis, BH Derkx, MA.Benninga,"Health related quality of life in disorders of defecation: the Defecation Disorder List”, Archives of Disease in Childhood,Vol.89, pp.1124-1127, 2004.

[3] M.H.Lu, "Defecation is detoxification”, Oriental medicated diet, Vol.2, pp.70, 2015.

[4] Y.F.Hong, "Bowel disease is pathogenic, defecation is also a technical activity”, Journal of self-care, Vol.4, pp.21, 2017.

[5] Y.Zhang, "Design and Implementation of Medical Monitor Based on Embedded System”, Tianjin University, 2012.

[6] L,Wu, “A weight weighted chair”, chinses utility model patent, No,CN201120069465, 2011.

[7] X.P.Liu, J.H.Liu, "Design and implementation of simple electronic scale based on MCU”, Computer Knowledge and Technology, Vol.12, pp.255-256, 2016.

[8] Z. Yanlin, X. Kaifeng. "Application of Cloud Storage in Hospital Information”, Chinese Medical Equipment Journal, Vol.33, pp.40-41., 2012. 
[9] Z.Q.Wang, "Unattended Hospital Wards Monitoring System Based on Wireless Radio Frequency Technology”, Modern Electronics Technique, Vol, 7, pp.159-161, 2010.

[10] M.J.Liu, Q.Zhang, Y.W.Mu, "Design of high precision electronic scale based on HX711”, Information \& Communications Vol.1, pp.142-144, 2017.

[11] Y.l. Zhang., K.F.Xu, "Application of Cloud Storage in Hospital Information”. Chinese Medical Equipment Journal, vol.33, pp.40-41, 2012. 